\title{
EI Sector Agrícola en Ecuador: Análisis de Correlación entre Utilidad, Participación de Mercado y Estructura de Capital
}

\author{
The Agricultural Sector in Ecuador: Correlation Analysis between Utility, Market \\ Share and Capital Structure
}

\author{
Patricio Arévalo-Chávez \\ Universidad Tecnológica Indoamérica \\ patricioarevalo@uti.edu.ec \\ Freddy Arévalo-Chávez \\ Universidad Central del Ecuador \\ fca.soporte@uce.edu.ec \\ Jorge Guadalupe-Lanas \\ Universidad Tecnológica Indoamérica \\ jorgeguadalupe@uti.edu.ec \\ Andrés Palacio-Fierro \\ Universidad Tecnológica Indoamérica \\ andrespalacio@uti.edu.ec
}

\section{RESUMEN}

La investigación que se presenta en el presente artículo tiene como objetivo relacionar tres grupos de variables: utilidad per cápita, participación de mercado y estructura de capital per cápita; con el fin de mostrar la correlación de cada una de estas variables en 17 industrias del sector agrícola ecuatoriano.

Los datos que se utilizaron para el análisis de esta correlación fueron los publicados por el Instituto Nacional de Estadísticas y Censos INEC, correspondientes al último Censo Nacional Económico, realizado en el año 2010.

Los resultados muestran que existen correlaciones significativas a niveles de $1 \%$ y $5 \%$, entre las variables: utilidad per cápita y participación de mercado, y entre las variables: utilidad per cápita y estructura de capital per cápita, en los sectores de cultivo de cereales (excepto arroz), legumbres y semillas oleaginosas, cultivos de arroz, cultivo de frutas tropicales y subtropicales, cultivo de frutas con hueso y con pepa y cultivo de especias y de plantas aromáticas, medicinales y farmacéuticas.

Palabras clave: oligopolio, sector agrícola, correlación, utilidad.

\begin{abstract}
The research showed in this article has the objective to relate three groups of variables: per capita profit, market share and per capita capital structure; in order to display the correlation of each of these variables in 17 industries of the Ecuadorian agricultural sector.

The data used for the analysis of this correlation were those published by the National Institute of Statistics and Censuses INEC, corresponding to the last National Economic Census, carried out in 2010.

The results show that there are significant correlations at levels of $1 \%$ and $5 \%$, among the next variables: per capita profit and market share, additionally the variables per capita income and per capita capital structure, in the sectors of cereal cultivation (except rice), legumes and oilseeds, rice
\end{abstract}


crops, tropical and subtropical fruit cultivation, fruit growing with bone and seed, and cultivation of spices and aromatic, medicinal and pharmaceutical plants.

Key words: oligopoly, agricultural sector, correlation, profit.

\section{INTRODUCCIÓN}

La producción agrícola en el país destinada al consumo interno es un producto que se encajaría mejor con la palabra en inglés commodity, es decir producto no diferenciado, lo que implica que los productores no tengan capacidad de negociación con sus compradores y que se refleja en la incapacidad de establecer el precio de venta de sus productos. Los precios de venta al consumidor finales son establecidos mediante una lista de precios referenciales como es el caso del banano, café y cacao (Latorre, Farrell, \& Martínez-Alier, 2015).

Además de que los agricultores no puedan negociar sus precios por estar ya establecidos en el mercado, tiene este sector varias barrera de entrada, como las altas inversiones iniciales para empezar cualquier actividad agrícola, los impredecibles cambio en el clima y fenómenos climáticos, así como el tiempo necesario entre la siembra y cosecha, junto a la estacionalidad de ciertos cultivos (Hassink, Hulsink, \& Grin, 2016).

En el caso de las empresas pequeñas y medianas se complica aún más el panorama, porque no poseen canales de distribución propios y su poder de negociación es casi nulo con los pocos compradores del sector, es decir con empresas agroindustriales, exportadores y comercializadores nacionales (Colloredo-Mansfeld, Ordoñez, \& Paltán López, 2016).

Al tener pocos compradores, las actividades agrícolas en Ecuador muestran estructuras de oligopsonio y también se aprecia una competencia monopolística a la vez, entre las principales empresas en este sector se tiene a las siguientes:

- Provefrut la cual es una empresa que exporta más de 300 marcas de brócoli y coliflor alrededor del mundo, especialmente a los Estados Unidos y al mercado japonés. Los empleados de esta empresa son mujeres. Provefrut tienen su propio laboratorio de biotecnología y producen bio-insumos que sustituyen a los agroquímicos. Unas 1.000 personas trabajan en el campo y otras 1.200 en las instalaciones de Provefrut S.A., donde se procesa el producto que envían a mercados de Europa, Japón y Estados Unidos (Comercio, 2017).

- La productora de alimentos Pronaca que absorbe el 70\% de la producción de maíz, genera más de 7.700 puestos de trabajo, ocupando el tercer lugar en emplear personas entre las empresas privadas. Pronaca trabaja con agricultores de Guayas y Los Ríos a quienes asesora en técnicas de cultivos y provee de los insumos y semillas necesarios para su proceso productivo. Por este tipo de relación y ayuda, los agricultores de la zona entregan su producción de manera directa a la empresa, con lo que se convierten en sus socios 
productivos, por este motivo, los productores pierden poder de negociación con ellos y están sujetos a imposición de precios en la venta de sus productos (Aguayo, López, \& Núñez, 2017).

- Los cultivos de trigo y maíz son procesados por las empresas de molienda de grados. Molinos Poultier ubicada en la ciudad de Latacunga, es una de las empresas molineras que dinamiza la economía del sector agrícola en la sierra centro del país, caracterizado por ser una provincia eminentemente agrícola. La compañía registró ventas en el año 2014 por un valor de USD 14,7 millones. En el 'ranking' empresarial de la Superintendencia de Compañías, constituye como una de las más importantes de la provincia de Cotopaxi (Ramirez, 2015).

- Una de las principales empresas avícolas que utiliza productos procesados de la molienda de granos, es Avitalsa que junto a Pronaca, Grupo Oro y otras, produce pavos para el mercado nacional (Agromar, 2009).

En lo que respecta los productos agrícolas el arroz constituye uno de los principales productos más demandados a nivel mundial (Mendez, 2017). En cuanto a la producción de arroz en el Ecuador es absorbida por 3 piladoras. La Corporación de Industriales Arroceros del Ecuador (Corpcom) agrupa a 42 piladoras a nivel nacional, las que atraen el 60\% del mercado. En invierno, la cosecha promedio de arroz en el país es de 440000 toneladas. Según la Corpcom en el 2014, hubo un déficit de $26 \%$ en la producción de arroz, debido al exceso de lluvias y a las plagas. Debido a la esta escasez hubo un proceso especulativo en los precios del arroz en el 2015 (Paspuel, 2015).

Otra importante producción es la de la caña de azúcar en el Ecuador, especialmente por parte de los ingenios privados, quienes han realizado en los últimos años una inversión constante en programas de investigación y desarrollo, además han producido nuevas variedades que son más resistentes a las plagas. Esta inversión ha llevado al sector a incrementar su productividad de 75 toneladas por hectárea hace 10 años, a las 90 por hectárea, que es el promedio actual. De manera paralela, estas trabajan juntamente con el Gobierno para la producción de la gasolina "ecopaís" que contiene un 5\% de etanol (derivado de la caña de azúcar); con este plan se pretende reemplazar a la gasolina extra que actualmente se vende. En Ecuador. Para el año 2015, la producción de etanol fue de 30 millones de litros anuales, que se irán incrementando hasta alcanzar los 100 millones, gracias a las inversiones que están realizando el gobierno juntamente con los tres principales ingenios del país (Tapia E. , 2015).

El sector agrícola analizado en el presente estudio se tomará en cuenta los subsectores a un nivel de 4 dígitos de la Clasificación Industrial Internacional Uniforme (CIIU) de las Naciones Unidas, la cual divide y clasifica a los sectores económicos. Cabe mencionar que el INEC aplicó la mencionada clasificación para toda actividad económica en el Ecuador. Ver en la tabla 1 se encuentran subcategorías del sector agrícola tomados en cuenta para este estudio con su correspondiente código y número de empresas analizadas. 
Por último, aquí se explica las variables a utilizar en este estudio: utilidad, la cual es el dinero que efectivamente podrían repartirse los dueños de las empresas después de un período económico, y estructura de capital que en esencia son los pasivos y patrimonio de una empresa, sin embargo, no serán tomados los valores totales sino per cápita, es decir por cabeza, por que se acerca más a la realidad de cada empresa, en lugar de tomar datos globales de todo el conjunto de cada sector. La última variable, y no menos importante, que se utilizará es la participación de mercado, la misma que indica la porción de ventas de cada empresa dentro del total de ventas dentro de su sector y mercado específicos.

\section{Tabla 1}

Sector agrícola: Número de empresas e industrias analizadas

\begin{tabular}{|c|c|c|}
\hline CIIU4 & Nombre de la industria & $\begin{array}{l}\text { Número de } \\
\text { empresas }\end{array}$ \\
\hline A0111 & Cultivo de cereales (excepto arroz), legumbres y semillas oleaginosas & 18 \\
\hline A0112 & Cultivo de arroz & 11 \\
\hline A0113 & Cultivo de hortalizas y melones, raíces y tubérculos & 155 \\
\hline A0114 & Cultivo de caña de azúcar & 6 \\
\hline A0115 & Cultivo de tabaco & 1 \\
\hline A0116 & Cultivo de plantas de fibras & 1 \\
\hline A0119 & Cultivo de otras plantas no perennes & 78 \\
\hline A0121 & Cultivo de uvas & 0 \\
\hline A0122 & Cultivo de frutas tropicales y subtropicales & 139 \\
\hline A0123 & Cultivo de cítricos & 12 \\
\hline A0124 & Cultivo de frutas con hueso y con pepa & 2 \\
\hline A0125 & Cultivo de otros frutos y nueces de árboles y arbustos & 23 \\
\hline A0126 & Cultivo de frutos oleaginosos & 7 \\
\hline A0127 & Cultivo de plantas con las que se preparan bebidas & 135 \\
\hline A0128 & Cultivo de especias y de plantas aromáticas, medicinales y farmacéuticas & 9 \\
\hline A0129 & Cultivo de otras plantas perennes & 2 \\
\hline A0130 & Propagación de plantas & 53 \\
\hline
\end{tabular}

Fuente: Censo Económico 2010. Instituto Nacional de Estadísticas y Censos INEC

Con estos antecedentes, se plantea la siguiente pregunta de investigación: ¿Existe una asociación significativa entre las variables utilidad per cápita, participación de mercado y estructura de capital per cápita en el sector agrícola del país y las industrias que la conforman?

La hipótesis de investigación que responde a esta pregunta es la siguiente: 
Existe una asociación significativa y positiva entre las variables: utilidad per cápita, participación de mercado y estructura de capital per cápita, en las industrias: A0111, A0112, A0113, A0114, A0115, A0116, A0119, A0121, A0122, A0123, A0124, A0125, A0126, A0127, A0128, A0129, A0130, que son parte del sector A01: Agricultura, Ganadería, Caza y Actividades de Servicios Conexas.

\section{MATERIALES Y MÉTODOS / METODOLOGÍA}

La investigación se realizó tomando como fuente de datos, las variables reportadas por el Instituto Nacional de Estadísticas y Censos INEC y proporcionadas por el último censo nacional económico realizado en el año 2010. Las variables que se calcularon fueron: la utilidad per cápita, participación de mercado y estructura de capital per cápita. Se eligió al sector A01: Agricultura, Ganadería, Caza y Actividades de Servicios Conexas, y se escogieron a 17 industrias relacionadas con el sector agrícola que se encuentran la tabla 1.

En la tabla 2, que se encuentra a continuación, se indica el número de establecimientos asociados a cada una de las industrias seleccionadas para el estudio y aquellos datos que fueron eliminados antes de su ingreso y cálculo por medio del programa estadístico SPSS.

\section{Tabla 2}

Número de establecimientos procesados y eliminados de la base de datos en cada una de las industrias del sector agrícola

\begin{tabular}{|l|l|c|c|}
\hline CIIU4 & \multicolumn{1}{|c|}{ Industria } & Número & Eliminados \\
\hline A0111 & $\begin{array}{l}\text { Cultivo de cereales (excepto arroz), legumbres y semillas } \\
\text { oleaginosas }\end{array}$ & 18 & 1 \\
\hline A0112 & Cultivo de arroz & 11 & 0 \\
\hline A0113 & Cultivo de hortalizas y melones, raíces y tubérculos & 155 & 0 \\
\hline A0114 & Cultivo de caña de azúcar & 6 & 0 \\
\hline A0115 & Cultivo de tabaco & 1 & 0 \\
\hline A0116 & Cultivo de plantas de fibras & 1 & 0 \\
\hline A0119 & Cultivo de otras plantas no perennes & 78 & 4 \\
\hline A0121 & Cultivo de uvas & 139 & 0 \\
\hline A0122 & Cultivo de frutas tropicales y subtropicales & 12 & 1 \\
\hline A0123 & Cultivo de cítricos & 2 & 0 \\
\hline A0124 & Cultivo de frutas con hueso y con pepa & 7 & 0 \\
\hline A0125 & Cultivo de otros frutos y nueces de árboles y arbustos & 35 & 0 \\
\hline A0126 & Cultivo de frutos oleaginosos & 9 & 0 \\
\hline A0127 & Cultivo de plantas con las que se preparan bebidas & & 0 \\
\hline & Cultivo de especias y de plantas aromáticas, medicinales y \\
A0128 & farmacéuticas & & 0 \\
\hline
\end{tabular}




\begin{tabular}{|c|l|c|c|} 
A0129 & Cultivo de otras plantas perennes & 2 & 1 \\
\hline A0130 & Propagación de plantas & 53 & 3 \\
\hline Total & & 552 & 14 \\
\hline
\end{tabular}

Fuente: Censo Económico 2010. Instituto Nacional de Estadísticas y Censos INEC

El criterio que se utilizó para eliminar algunos establecimientos económicos de la base de datos procesada fue la de 'ingresos cero', es decir aquellos que de acuerdo al INEN reportaron cero ingresos.

Cabe señalar que se escogió al sector agrícola por ser uno de los más importantes sectores que contribuyen al producto interno bruto del país con un aporte relativo que supera el $9 \%$, además es un sector intensivo en mano de obra y de inversión en tecnología (Izquierdo, 2017).

Adicionalmente, por un parte, para las variables: utilidad per cápita, participación de mercado y estructura de capital per cápita se tomó en cuenta los siguientes criterios:

- Para el cálculo de la utilidad, se utilizó las variables: ingresos y gastos, proporcionadas por el censo nacional económico, tomando en cuenta el número de empleados totales que laboran en cada establecimiento económico.

- El cálculo de la variable: participación de mercado, tomó en cuenta todos los ingresos percibidos por el establecimiento económico y su participación en los ingresos totales de la industria analizada.

- Para la variable estructura de capital se tomó en cuenta todos los activos fijos con una duración superior a un año y que son utilizados para la gestión de la organización.

- En la variable ingresos se consideró el total de ingresos por las ventas de sus productos, bienes o servicios prestados que tuvo el establecimiento en el transcurso del año 2009. El criterio utilizado para el registro de esta variable fueron las entradas por concepto de las actividades primarias del establecimiento, es decir aquellas relacionadas con el giro del negocio (Asencio Ochoa, 2017).

Por otra parte, el personal técnico del INEC realizó capacitaciones continuas a los empadronadores, con el fin de que en el momento de registro de esta variable tomen en cuenta las siguientes definiciones:

- Ventas de productos elaborados: ingresos por la venta de bienes fabricados o manufacturados por el establecimiento (INEC, 2017).

- Venta de mercaderías: ingresos por la venta de bienes sin transformación, es decir aquellos que se vendieron en las mismas condiciones en que los que fueron comprados (INEC, 2017).

- Ingresos por prestación de servicios: ingresos por concepto de prestación de servicios, como: servicios de consultoría, honorarios profesionales, etc. (INEC, 2017). 
De esta forma, todos los ingresos adicionales y extraordinarios que no son producto de las actividades primarias del establecimiento también fueron tomados en cuenta para el cálculo de los ingresos.

Consecuentemente es importante destacar que de la variable: "ingreso" se tomó las siguientes variables del Censo Nacional Económico 2010 que se encuentran en la tabla 3.

Tabla 3

Códigos de la variable ingreso

\begin{tabular}{|l|l|}
\hline \multicolumn{1}{|c|}{ Códigos } & \multicolumn{1}{c|}{ Definición } \\
\hline Ingresos & Total de ingresos anuales percibidos por ventas o prestación de servicios \\
\hline otr_ing & Otros ingresos anuales \\
\hline ing_ext & Ingresos extraordinarios anuales \\
\hline
\end{tabular}

Fuente: Censo Económico 2010. Instituto Nacional de Estadísticas y Censos INEC

\section{Variable gastos}

Gastos en remuneraciones: Todos los pagos en dinero o en especies efectuados por el establecimiento, por los servicios recibidos por los empleados que trabajan bajo relación de dependencia y trabajos esporádicos realizados por el establecimiento para cumplir con sus obligaciones (INEC, 2017).

Gastos anuales en materias primas y materias auxiliares: Valor pagado por concepto de todos los insumos utilizados por el establecimiento en el proceso productivo relacionadas con empresas de: manufactura, servicios de restaurantes y agricultura (INEC, 2017).

Gastos anuales en repuestos y accesorios: Gasto en repuestos y accesorios incurridos por el establecimiento para extender la vida útil o el aumento de la capacidad productiva de sus activos productivos (INEC, 2017).

Gastos anuales en envases y embalajes: Valores pagados por el consumo y utilización de envases y embalajes, utilizados tanto para la prestación de servicios, como para la comercialización de productos (INEC, 2017).

Gastos anuales en compras y mercaderías: Valor pagado por el establecimiento por concepto de la compra de mercadería y que se destinó para la venta sin proceso de transformación (INEC, 2017).

Gastos por servicios prestados por terceros y alquileres: Gastos por concepto de honorarios profesionales, servicios informáticos, investigación y desarrollo, servicios jurídicos, publicidad, etc. (INEC, 2017).

Otros egresos anuales corrientes: Gastos que no corresponden a los detallados anteriormente, como: pagos de servicios básicos, teléfonos, seguros, materiales de oficina, etc. (INEC, 2017). 
En la tabla número 4 se indica el código de variable y la descripción de cada una de las variables que se tomó en cuenta como "costo -gasto".

Tabla 4

Códigos de la variable costos y gastos

\begin{tabular}{|l|l|}
\hline \multicolumn{1}{|c|}{ Códigos } & \multicolumn{1}{c|}{ Definición } \\
\hline gast_rem & Gastos anuales en remuneraciones \\
\hline gast_mat & Gastos anuales en materia prima \\
\hline gast_rac & Gastos anuales en repuestos y accesorios \\
\hline gast_ee & Gastos anuales en envases y embalajes \\
\hline gast_com & Gastos anuales en compras y mercaderías \\
\hline gast_ter & Gastos anuales por servicios prestados por terceros y alquileres \\
\hline Egresos & Otros egresos anuales corrientes \\
\hline
\end{tabular}

Fuente: Censo Económico 2010. Instituto Nacional de Estadísticas y Censos INEC

\section{Variable estructura de capital}

Compras de activos fijos existencias al 31 de diciembre: Son todos los bienes físicos de posesión del establecimiento que se los utiliza para gestión de la organización y poseen una duración mayor a un año que no están sometidos a actividades comerciales corrientes. Ejemplo: edificios, vehículos, terrenos, instalaciones, maquinaria, equipos de oficina, etc. (INEC, 2017).

Valor de activos fijos existencias al 31 de diciembre: Valor de los activos fijos que tuvo el establecimiento al 1 de enero y al 31 de diciembre del 2009. En establecimientos pequeños estos activos son: herramientas pequeñas con una durabilidad superior a un año (taladros, serruchos, cepillos de carpintería, etc.), mesas, vitrinas, refrigeradoras, cocinas, ventiladores, máquinas de coser, tornos, etc. (INEC, 2017).

En la tabla 5 se indica las variables consideradas para el cálculo de la estructura de capital per cápita.

Tabla 5

Códigos de la variable valor de activos fijos

\begin{tabular}{|l|l|}
\hline \multicolumn{1}{|c|}{ Códigos } & \multicolumn{1}{c|}{ Definición } \\
\hline s5p7_1c1 & Compras de activos fijos existencias al 31 de diciembre \\
\hline s5p7_4c2 & Valor de activos fijos existencias al 31 de diciembre \\
\hline
\end{tabular}


Fuente: Censo Económico 2010. Instituto Nacional de Estadísticas y Censos INEC

En base a estos 3 grupos de variables, posteriormente se procedió a realizar el cálculo las variables: utilidad per cápita, participación de mercado y estructura de capital per cápita.

\section{RESULTADOS}

Una vez calculadas las variables: utilidad per cápita, participación de mercado y estructura de capital per cápita, se procedió a ingresarlas en el programa SPSS, y determinar las correlaciones existentes entre dichas variables.

Los resultados de las correlaciones cruzadas entre las variables indicadas se muestran en el anexo 1. Sector Agrícola: Análisis de correlación entre las variables: utilidad per cápita, participación de mercado y estructura de capital per cápita. De los sectores analizados, se encontró:

- Correlación significativa en el nivel $1 \%$ entre las variables: utilidad per cápita y participación de mercado, y entre las variables utilidad per cápita y estructura de capital per cápita en los sectores: A0111: cultivo de cereales (excepto arroz), legumbres y semillas oleaginosas, A0112: cultivos de arroz, A0122: cultivo de frutas tropicales y subtropicales, A0124: cultivo de frutas con hueso y con pepa y A0128: cultivo de especias y de plantas aromáticas, medicinales y farmacéuticas.

- Correlación significativa al $1 \%$ entre las variables: participación de mercado y estructura de capital per cápita, en los sectores: A0111: cultivo de cereales (excepto arroz), legumbres y semillas oleaginosas y A0114: cultivo de caña de azúcar.

- Correlación significativa en el nivel 0,05, entre las variables: estructura de capital per cápita y utilidad per cápita, en los sectores: A0112: cultivo de arroz, A0125: cultivo de otros frutos y nueces de árboles y arbustos y A0130: propagación de plantas.

- Correlación significativa al $1 \%$ entre las variables: estructura de capital per cápita y utilidad per cápita, en el sector: A0124: cultivo de frutas con hueso y con pepa.

- Correlación significativa al nivel $5 \%$ entre las variables: participación de mercado y estructura de capital per cápita, en el sector: A0122: cultivo de frutas tropicales y subtropicales.

- Correlación no significativa entre las variables: utilidad per cápita y estructura de capital per cápita en las industrias: A0113: cultivo de hortalizas y melones, raíces y tubérculos, A0119: cultivo de otras plantas no perennes, A0123: cultivo de cítricos, A0126: cultivo de frutos oleaginosos, A0127: cultivo de plantas con las que se preparan bebidas y A0130: propagación de plantas.

- Correlación no significativa entre las variables: utilidad per cápita y participación de mercado en las industrias: A0113: cultivo de hortalizas y melones, raíces y tubérculos, 
A0119: cultivo de otras plantas no perennes, A0123: cultivo de cítricos, A0125: cultivo de otros frutos y nueces de árboles y arbustos, A0126: cultivo de frutos oleaginosos y A0127: cultivo de plantas con las que se preparan bebidas.

- Correlación no significativa entre las variables: participación de mercado y estructura de capital per cápita en las industrias: A0113: cultivo de hortalizas y melones, raíces y tubérculos, A0119: cultivo de otras plantas no perennes, A0123: cultivo de cítricos, A0125: cultivo de otros frutos y nueces de árboles y arbustos, A0126: cultivo de frutos oleaginosos, A0127: cultivo de plantas con las que se preparan bebidas y A0130: propagación de plantas.

\section{CONCLUSIONES}

Los hallazgos muestran correlaciones significativas en 6 de las 17 industrias analizadas. Estas correlaciones se evidencian de manera clara en la utilidad per cápita y la participación de mercado, para las industrias de cultivo de cereales (excepto arroz), las legumbres y semillas oleaginosas, cultivo de arroz, cultivo de frutas tropicales y subtropicales, y propagación de plantas. Además de la correlación entre la utilidad per cápita y la estructura de capital per cápita entre las industrias del cultivo de cereales (excepto arroz), legumbres y semillas oleaginosas, cultivo de arroz, cultivo de frutas tropicales y subtropicales.

Estos hallazgos dan cuenta de la dinámica que interrelaciona de manera significativa a esas seis industrias con las tres variables mencionadas, abriendo así la posibilidad de profundizar a través de un estudio econométrico las relaciones de causalidad que determinen resultados intra-sectoriales necesarios para acompañar los cambios productivos en el Ecuador.

\section{AGRADECIMIENTOS}

El presente trabajo de investigación no habría sido posible sin el apoyo económico y financiero de la Universidad Tecnológica Indoamérica, además del aporte técnico y de conocimientos de todos los integrantes del equipo de trabajo. 


\section{REFERENCIAS}

1. Agromar. (21 de noviembre de 2009). El Comercio. Obtenido de La producción de carne de pavo se incrementó este año: http://www.elcomercio.com/actualidad/produccion-carne-pavoincremento-este.html

2. Aguayo, N., López, V., \& Núñez, T. (2017). Cultura y Gestión del Conocimiento en organizaciones del Ecuador. Revista Publicando, 4(11 (2)), 70-83.

3. Asencio Ochoa, P. A. (2017). Los proyectos de inversión en el desarrollo del sector agropecuaario del Ecuador. Machala - Ecuador: Unidad Académica de Ciencias Agropecuarias.

4. Colloredo-Mansfeld, R., Ordoñez, A., \& Paltán López, H. (2016). Conflicts, Territories, and the Institutionalization of Post-Agrarian Economies on an Expanding Tourist Frontier in Quilotoa, Ecuador. World Development.

5. Comercio, E. (2 de Julio de 2017). Los productos de exportación suman kilogramos de CO2. El Comercio.

6. Hassink, J., Hulsink, W., \& Grin, J. (2016). Entrepreneurship in agriculture and healthcare: Different entry strategies of care farmers. Journal of Rural Studies, 27-39.

7. INEC. (5 de Junio de 2017). Censo Nacional Económico 2010. Obtenido de ecuadorencifras: http://www.ecuadorencifras.gob.ec/wpcontent/descargas/Estadisticas_economicas/descarga_INEC/Metodologia.pdf

8. Izquierdo, O. (2017). El sector agrícola en el TLC y sus consecuencias para el Ecuador. Revista AFESE, 43(43).

9. Latorre, S., Farrell, K., \& Martínez-Alier, J. (2015). The commodification of nature and socioenvironmental resistance in Ecuador: An inventory of accumulation by dispossession cases, 1980-2013. Ecological Economics, 116, 58-69.

10. Mendez, P. (Marzo de 2017). Infoarroz. Obtenido de Informativo mensual del mercado mundial del arroz: http://www.infoarroz.org/portal/uploadfiles/20170403193421_15_ia0317es.pdf

11. Paspuel, W. (4 de abril de 2015). El Comercio. Obtenido de Las reservas de arroz se agotan en las piladoras: http://www.elcomercio.com/actualidad/deficit-arroz-piladoras.html

12. Ramirez, S. (21 de noviembre de 2015). El Comercio. Obtenido de Molinos Poultier resistió la fuerza del Cotopaxi y el cambio de dueños: http://www.elcomercio.com/actualidad/molinospoultier-volcancotopaxi-latacungaproduccion.html

13. Tapia, E. (15 de septiembre de 2015). El Comercio. Obtenido de El fenómeno de El Niño mantiene en alerta al sector cañicultor: http://www.elcomercio.com/actualidad/guayaquilfenomenoelnino-sectorcanicultor.html 
Anexo 1.

Sector agrícola: análisis de correlación entre las variables: utilidad per cápita, participación de mercado y estructura de capital per cápita

\begin{tabular}{|c|c|c|c|c|c|}
\hline Industria & Variables & Tipo de correlación & uti_per & part_mer & est_caper \\
\hline \multirow{3}{*}{$\begin{array}{l}\mathrm{A} 0111 \\
\mathrm{~N}=17\end{array}$} & uti_per & $\begin{array}{l}\text { Correlación de Pearson } \\
\text { Sig. (bilateral) }\end{array}$ & 1 & $\begin{array}{c}943^{* *} \\
0\end{array}$ & $\begin{array}{c}819 * * \\
0\end{array}$ \\
\hline & part_mer & $\begin{array}{l}\text { Correlación de Pearson } \\
\text { Sig. (bilateral) }\end{array}$ & $\begin{array}{c}943^{* *} \\
0\end{array}$ & 1 & $\begin{array}{l}, 623 * * \\
0,008\end{array}$ \\
\hline & est_caper & $\begin{array}{l}\text { Correlación de Pearson } \\
\text { Sig. (bilateral) }\end{array}$ & $\begin{array}{c}, 819 * * \\
0\end{array}$ & $\begin{array}{l}623 * * \\
0,008\end{array}$ & 1 \\
\hline \multirow{3}{*}{$\begin{array}{l}\mathrm{A} 0112 \\
\mathrm{~N}=11\end{array}$} & uti_per & $\begin{array}{l}\text { Correlación de Pearson } \\
\text { Sig. (bilateral) }\end{array}$ & 1 & $\begin{array}{l}, 805^{* *} \\
0,003\end{array}$ & $\begin{array}{l}656^{*} \\
0,028\end{array}$ \\
\hline & part_mer & $\begin{array}{l}\text { Correlación de Pearson } \\
\text { Sig. (bilateral) }\end{array}$ & $\begin{array}{l}805^{* *} \\
0,003\end{array}$ & 1 & $\begin{array}{c}927^{* *} \\
0\end{array}$ \\
\hline & est_caper & $\begin{array}{l}\text { Correlación de Pearson } \\
\text { Sig. (bilateral) }\end{array}$ & $\begin{array}{l}, 656^{*} \\
0,028\end{array}$ & $\begin{array}{c}927 * * \\
0\end{array}$ & 1 \\
\hline \multirow{3}{*}{$\begin{array}{l}A 0113 \\
N=155\end{array}$} & uti_per & $\begin{array}{l}\text { Correlación de Pearson } \\
\text { Sig. (bilateral) }\end{array}$ & 1 & $\begin{array}{l}0,069 \\
0,392\end{array}$ & $\begin{array}{l}0,114 \\
0,158\end{array}$ \\
\hline & part_mer & $\begin{array}{l}\text { Correlación de Pearson } \\
\text { Sig. (bilateral) }\end{array}$ & $\begin{array}{l}0,069 \\
0,392\end{array}$ & 1 & $\begin{array}{l}-0,001 \\
0,988\end{array}$ \\
\hline & est_caper & $\begin{array}{l}\text { Correlación de Pearson } \\
\text { Sig. (bilateral) }\end{array}$ & $\begin{array}{l}0,114 \\
0,158\end{array}$ & $\begin{array}{l}-0,001 \\
0,988\end{array}$ & 1 \\
\hline \multirow{3}{*}{$\begin{array}{l}\mathrm{A} 0114 \\
\mathrm{~N}=6\end{array}$} & uti_per & $\begin{array}{l}\text { Correlación de Pearson } \\
\text { Sig. (bilateral) }\end{array}$ & 1 & $\begin{array}{l}-0,137 \\
0,796\end{array}$ & $\begin{array}{l}955^{* *} \\
0,003\end{array}$ \\
\hline & part_mer & $\begin{array}{l}\text { Correlación de Pearson } \\
\text { Sig. (bilateral) }\end{array}$ & $\begin{array}{l}-0,137 \\
0,796\end{array}$ & 1 & $\begin{array}{l}-0,118 \\
0,823\end{array}$ \\
\hline & est_caper & $\begin{array}{l}\text { Correlación de Pearson } \\
\text { Sig. (bilateral) }\end{array}$ & $\begin{array}{l}, 955^{* *} \\
0,003\end{array}$ & $\begin{array}{l}-0,118 \\
0,823\end{array}$ & 1 \\
\hline \multirow{3}{*}{$\begin{array}{l}\text { A0119 } \\
\mathrm{N}=74\end{array}$} & uti_per & $\begin{array}{l}\text { Correlación de Pearson } \\
\text { Sig. (bilateral) }\end{array}$ & 1 & $\begin{array}{l}0,092 \\
0,438\end{array}$ & $\begin{array}{l}0,081 \\
0,495\end{array}$ \\
\hline & part_mer & $\begin{array}{l}\text { Correlación de Pearson } \\
\text { Sig. (bilateral) }\end{array}$ & $\begin{array}{l}0,092 \\
0,438\end{array}$ & 1 & $\begin{array}{l}-0,051 \\
0,666\end{array}$ \\
\hline & est_caper & $\begin{array}{l}\text { Correlación de Pearson } \\
\text { Sig. (bilateral) }\end{array}$ & $\begin{array}{l}0,081 \\
0,495\end{array}$ & $\begin{array}{l}-0,051 \\
0,666\end{array}$ & 1 \\
\hline
\end{tabular}




\begin{tabular}{|c|c|c|c|c|c|}
\hline \multirow{3}{*}{$\begin{array}{l}\mathrm{A} 0122 \\
\mathrm{~N}=135\end{array}$} & uti_per & $\begin{array}{l}\text { Correlación de Pearson } \\
\text { Sig. (bilateral) }\end{array}$ & 1 & $\begin{array}{l}, 212 * \\
0,014\end{array}$ & $\begin{array}{l}, 271 * * \\
0,001\end{array}$ \\
\hline & part_mer & $\begin{array}{l}\text { Correlación de Pearson } \\
\text { Sig. (bilateral) }\end{array}$ & $\begin{array}{l}, 212 * \\
0,014\end{array}$ & 1 & $\begin{array}{l}, 214^{*} \\
0,013\end{array}$ \\
\hline & est_caper & $\begin{array}{l}\text { Correlación de Pearson } \\
\text { Sig. (bilateral) }\end{array}$ & $\begin{array}{l}, 271 * * \\
0,001\end{array}$ & $\begin{array}{l}, 214^{*} \\
0,013\end{array}$ & 1 \\
\hline \multirow{3}{*}{$\begin{array}{l}\mathrm{A} 0123 \\
\mathrm{~N}=11\end{array}$} & uti_per & $\begin{array}{l}\text { Correlación de Pearson } \\
\text { Sig. (bilateral) }\end{array}$ & 1 & $\begin{array}{l}0,265 \\
0,432\end{array}$ & $\begin{array}{l}0,213 \\
0,529\end{array}$ \\
\hline & part_mer & $\begin{array}{l}\text { Correlación de Pearson } \\
\text { Sig. (bilateral) }\end{array}$ & $\begin{array}{l}0,265 \\
0,432\end{array}$ & 1 & $\begin{array}{l}0,059 \\
0,864\end{array}$ \\
\hline & est_caper & $\begin{array}{l}\text { Correlación de Pearson } \\
\text { Sig. (bilateral) }\end{array}$ & $\begin{array}{l}0,213 \\
0,529\end{array}$ & $\begin{array}{l}0,059 \\
0,864\end{array}$ & 1 \\
\hline Industria & Variables & Tipo de correlación & uti_per & part_mer & est_caper \\
\hline \multirow{3}{*}{$\begin{array}{l}\mathrm{A} 0124 \\
\mathrm{~N}=2\end{array}$} & uti_per & $\begin{array}{l}\text { Correlación de Pearson } \\
\text { Sig. (bilateral) }\end{array}$ & 1 & $\begin{array}{c}-1,000 * * \\
.\end{array}$ & $1,000 * *$ \\
\hline & part_mer & $\begin{array}{l}\text { Correlación de Pearson } \\
\text { Sig. (bilateral) }\end{array}$ & $\begin{array}{c}-1,000 * * \\
.\end{array}$ & 1 & $\begin{array}{c}-1,000 * * \\
.\end{array}$ \\
\hline & est_caper & $\begin{array}{l}\text { Correlación de Pearson } \\
\text { Sig. (bilateral) }\end{array}$ & $\begin{array}{c}1,000^{* *} \\
.\end{array}$ & $\begin{array}{c}-1,000 * * \\
.\end{array}$ & 1 \\
\hline \multirow{3}{*}{$\begin{array}{l}\mathrm{A} 0125 \\
\mathrm{~N}=23\end{array}$} & uti_per & $\begin{array}{l}\text { Correlación de Pearson } \\
\text { Sig. (bilateral) }\end{array}$ & 1 & $\begin{array}{l}0,368 \\
0,084\end{array}$ & $\begin{array}{l}, 458^{*} \\
0,028\end{array}$ \\
\hline & part_mer & $\begin{array}{l}\text { Correlación de Pearson } \\
\text { Sig. (bilateral) }\end{array}$ & $\begin{array}{l}0,368 \\
0,084\end{array}$ & 1 & $\begin{array}{c}0,082 \\
0,71\end{array}$ \\
\hline & est_caper & $\begin{array}{l}\text { Correlación de Pearson } \\
\text { Sig. (bilateral) }\end{array}$ & $\begin{array}{l}458 * \\
0,028\end{array}$ & $\begin{array}{c}0,082 \\
0,71\end{array}$ & 1 \\
\hline \multirow{3}{*}{$\begin{array}{l}\text { A0126 } \\
N=7\end{array}$} & uti_per & $\begin{array}{l}\text { Correlación de Pearson } \\
\text { Sig. (bilateral) }\end{array}$ & 1 & $\begin{array}{l}-0,19 \\
0,683\end{array}$ & $\begin{array}{l}0,015 \\
0,975\end{array}$ \\
\hline & part_mer & $\begin{array}{l}\text { Correlación de Pearson } \\
\text { Sig. (bilateral) }\end{array}$ & $\begin{array}{l}-0,19 \\
0,683\end{array}$ & 1 & $\begin{array}{l}-0,453 \\
0,308\end{array}$ \\
\hline & est_caper & $\begin{array}{l}\text { Correlación de Pearson } \\
\text { Sig. (bilateral) }\end{array}$ & $\begin{array}{l}0,015 \\
0,975\end{array}$ & $\begin{array}{l}-0,453 \\
0,308\end{array}$ & 1 \\
\hline \multirow{2}{*}{$\begin{array}{l}\mathrm{A} 0127 \\
\mathrm{~N}=35\end{array}$} & uti_per & $\begin{array}{l}\text { Correlación de Pearson } \\
\text { Sig. (bilateral) }\end{array}$ & 1 & $\begin{array}{l}0,101 \\
0,562\end{array}$ & $\begin{array}{l}0,012 \\
0,946\end{array}$ \\
\hline & part_mer & $\begin{array}{l}\text { Correlación de Pearson } \\
\text { Sig. (bilateral) }\end{array}$ & $\begin{array}{l}0,101 \\
0,562\end{array}$ & 1 & $\begin{array}{l}-0,001 \\
0,994\end{array}$ \\
\hline
\end{tabular}




\begin{tabular}{|c|c|c|c|c|c|}
\hline & est_caper & $\begin{array}{l}\text { Correlación de Pearson } \\
\text { Sig. (bilateral) }\end{array}$ & $\begin{array}{l}0,012 \\
0,946\end{array}$ & $\begin{array}{r}-0,001 \\
0,994\end{array}$ & 1 \\
\hline \multirow{3}{*}{$\begin{array}{l}\mathrm{A} 0128 \\
\mathrm{~N}=9\end{array}$} & uti_per & $\begin{array}{l}\text { Correlación de Pearson } \\
\text { Sig. (bilateral) }\end{array}$ & 1 & $\begin{array}{c}946 * * \\
0\end{array}$ & $\begin{array}{c}945^{* *} \\
0\end{array}$ \\
\hline & part_mer & $\begin{array}{l}\text { Correlación de Pearson } \\
\text { Sig. (bilateral) }\end{array}$ & $\begin{array}{c}946 * * \\
0\end{array}$ & 1 & $\begin{array}{c}1,000^{* *} \\
0\end{array}$ \\
\hline & est_caper & $\begin{array}{l}\text { Correlación de Pearson } \\
\text { Sig. (bilateral) }\end{array}$ & $\begin{array}{c}945 * * \\
0\end{array}$ & $\begin{array}{c}1,000^{* * *} \\
0\end{array}$ & 1 \\
\hline \multirow{3}{*}{$\begin{array}{l}A 0130 \\
N=50\end{array}$} & uti_per & $\begin{array}{l}\text { Correlación de Pearson } \\
\text { Sig. (bilateral) }\end{array}$ & 1 & $\begin{array}{l}-, 317 * \\
0,025\end{array}$ & $\begin{array}{c}0,01 \\
0,942\end{array}$ \\
\hline & part_mer & $\begin{array}{l}\text { Correlación de Pearson } \\
\text { Sig. (bilateral) }\end{array}$ & $\begin{array}{l}-317 * \\
0,025\end{array}$ & 1 & $\begin{array}{l}0,147 \\
0,308\end{array}$ \\
\hline & est_caper & $\begin{array}{l}\text { Correlación de Pearson } \\
\text { Sig. (bilateral) }\end{array}$ & $\begin{array}{c}0,01 \\
0,942\end{array}$ & $\begin{array}{l}0,147 \\
0,308\end{array}$ & 1 \\
\hline
\end{tabular}

** La correlación es significativa en el nivel 0,01 (2 colas).

* La correlación es significativa en el nivel 0,05 (2 colas). 